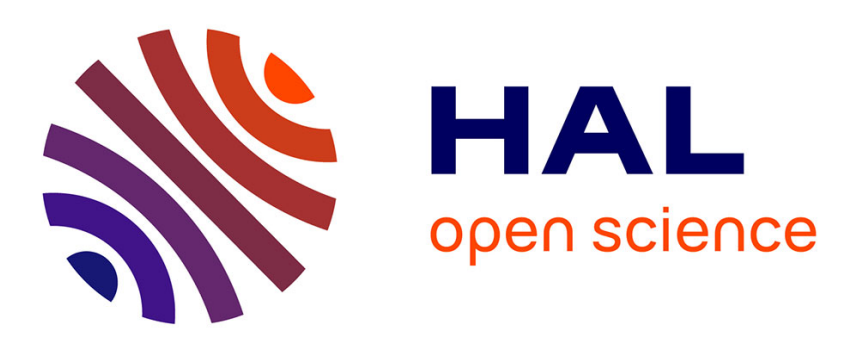

\title{
Universal highly efficient conditional knockout system in Leishmania, with a focus on untranscribed region preservation
}

Akila Yagoubat, Lucien Crobu, Laurence Berry, Nada Kuk, Michèle Lefebvre, Amélie Sarrazin, Patrick Bastien, Yvon Sterkers

\section{To cite this version:}

Akila Yagoubat, Lucien Crobu, Laurence Berry, Nada Kuk, Michèle Lefebvre, et al.. Universal highly efficient conditional knockout system in Leishmania, with a focus on untranscribed region preservation. Cellular Microbiology, inPress, 10.1111/cmi.13159 . hal-02499098

\section{HAL Id: hal-02499098 \\ https://hal.umontpellier.fr/hal-02499098}

Submitted on 9 Mar 2020

HAL is a multi-disciplinary open access archive for the deposit and dissemination of scientific research documents, whether they are published or not. The documents may come from teaching and research institutions in France or abroad, or from public or private research centers.
L'archive ouverte pluridisciplinaire HAL, est destinée au dépôt et à la diffusion de documents scientifiques de niveau recherche, publiés ou non, émanant des établissements d'enseignement et de recherche français ou étrangers, des laboratoires publics ou privés. 


\title{
Universal highly efficient conditional knockout system in Leishmania, with a focus on UTR preservation
}

\author{
Akila Yagoubat ${ }^{(1)}$, Lucien Crobu ${ }^{(1)}$, Laurence Berry ${ }^{(2)}$, Nada Kuk ${ }^{(1)}$, Michèle Lefebvre ${ }^{(1)}$, \\ Amélie Sarrazin ${ }^{(3)}$, Patrick Bastien ${ }^{(1)}$ and Yvon Sterkers ${ }^{(1)^{*}}$ \\ ${ }^{(1)}$ MiVEGEC, Univ. Montpellier, CNRS, IRD, CHU, Montpellier, France \\ ${ }^{(2)}$ LPHI - Laboratory of Pathogen Host Interactions \& MEA - Microscopie Electronique \\ Analytique, CNRS, Univ. Montpellier, Montpellier, France.
}

${ }^{(3)}$ Montpellier RIO Imaging Facility, Montpellier BIOCAMPUS, Univ. Montpellier, Arnaud de Villeneuve Campus Imaging Facility - Institut de Génétique Humaine-CNRS, Montpellier, France.

* To whom correspondence should be addressed

Postal address: Laboratoire de Parasitologie-Mycologie, CHU de Montpellier, 39, Avenue Charles Flahault, 34295 Montpellier cedex 5, France.

Telephone: +33 4676355 13, Fax: +33 4676323 58, Email: yvon.sterkers@ umontpellier.fr

This manuscript has been published, please cite: Yagoubat A, Crobu L, Berry L, Kuk N, Lefebvre M, Sarrazin A, Bastien P, Sterkers Y. Universal highly efficient conditional knockout system in Leishmania, with a focus on UTR preservation. Cell Microbiol. 2020 Jan 7:e13159. doi: 10.1111/cmi.13159.

\begin{abstract}
Trypanosomatids are divergent eukaryotes of high medical and economical relevance. Their biology exhibits original features which remain poorly understood; particularly Leishmania is known for its high degree of genomic plasticity which makes genomic manipulation challenging. CRISPR-Cas9 has been applied successfully to these parasites providing a robust tool to study non-essential gene functions. Here, we have developed a versatile inducible system combining Di-CRE recombinase and CRISPR-Cas9 advantages. Cas9 is used to integrate the LoxP sequences and the Cre-recombinase catalyzes the recombination between LoxP sites thereby excising the target gene. We used an L. mexicana cell line expressing DiCRE, Cas9 and T7 polymerase and then transfected donor DNAs and single guide RNAs as PCR products. Since the location of LoxP sequences in the genomic DNA can interfere with the function and localization of certain proteins of interest, we proposed to target the least transcribed regions upstream and/or downstream the gene of interest. To do so, we developed 'universal' template plasmids for donor DNA cassettes with or without a tag, where LoxP sequences may be located either immediately upstream the ATG and downstream the stop codon of the gene of interest, or in the least transcribed areas of intergenic regions. Our methodology is fast, PCR based (molecular cloning-free), highly efficient, versatile, and able to overcome the problems posed by genomic plasticity in Leishmania.
\end{abstract}




\section{Introduction}

Leishmania sp. are protozoan parasites belonging to the Kinetoplastid order which includes other human pathogens of medical importance such as Trypanosoma brucei, causing sleeping sickness, and T. cruzi, responsible for Chagas' disease. More than 20 Leishmania species can infect humans (Akhoundi et al., 2016) and they cause a wide spectrum of diseases ranging from self-healing cutaneous infections to a visceral form which is fatal if left untreated. Leishmaniasis spreads over 98 countries in five continents, including temperate climates, with a high incidence of almost 1 million new cases per year, and 26000 to 65000 deaths annually (WHO 2019). The current situation shows that both the incidence of the disease and drug resistance are increasing despite the availability of several molecules. The search for novel drug candidates and preventive strategies is therefore needed to reduce the importance of this neglected disease. Like many protozoa, Trypanosomatids are considered 'divergent eukaryotes' and, as such, exhibit a high number of original features in their cell and molecular biology. Leishmania is characterized by a particularly high degree of genome plasticity which appears to be genome-wide, and perhaps stochastic, including mosaic aneuploidy (Sterkers, Lachaud, Crobu, Bastien, \& Pagès, 2011) and gene amplification (Ubeda et al., 2008). While aneuploidy is deleterious in most eukaryotes (Iourov, Vorsanova, \& Yurov, 2008; Torres et al., 2007), Leishmania parasites are unique in their good tolerance to mosaic aneuploidy which may confer various advantages such as drug resistance and adaptability to the environment (Prieto Barja et al., 2017). However, this feature complicates the understanding of Leishmania biology and hinders attempts to its genetic manipulation. Moreover, while RNAinterference may be used in other parasites like T. brucei, enabling the characterization of essential genes, this machinery is absent in the subgenus Leishmania (Lye et al., 2010). In the recent years, the CRISPRCas9 technology has been established in Leishmania, allowing certain aspects of its biology to be addressed by facilitating a range of genetic manipulations which used to be challenging and time consuming (Sollelis et al., 2015; Zhang \& Matlashewski, 2015); (Zhang, Lypaczewski, \& Matlashewski, 2017). In 2017, Eva Gluenz's laboratory developed an improved CRISPR-Cas9 system for genome editing of Trypanosomatids (Beneke et al., 2017), based on transfections of PCR products for both the donor DNA and the sgRNA. This simplified the process of generating mutants without the need for molecular cloning, thus accelerating gene characterization in Leishmania. However, this system is not adapted to study genes essential to the parasite's survival. Several attempts to set up an inducible system for the knockout (iKO) of essential genes have been described (Yao, Luo, Hsiao, Donelson, \& Wilson, 2007); (Kraeva, Ishemgulova, Lukes, \& Yurchenko, 2014) but none is really satisfactory.

In this study, we propose a Rapamycin-DiCRE-based inducible CRISPR-Cas9 system to generate iKOs of essential genes in L. mexicana. LoxP sequences are introduced into all the target gene alleles using the Cas9 activity. The CRE recombinase is split into two inactive fragments, that are fused to FKBP12 and FRB domains. The coding sequences are integrated in the SSU of the L. mexicana strain (Damasceno, Tosi, Santos, \& Mottram, 2019). Addition of rapamycin in the culture medium leads to dimerization of the two enzyme fragments and the reconstitution of enzymatic activity. The active Cre recombinase catalyzes sitespecific DNA recombination between the two LoxP sites and excision of the target gene (Figure 1). We established an optimized DNA cloning-free approach 
combining Di-CRE and CRISPR-Cas9 advantages, based upon two 'universal' template plasmids allowing the preparation of the donor DNA cassettes by PCR. These plasmids are so-called 'universal' because they can be used to generate PCR donor DNA for any gene of interest (GOI) without the need for further molecular cloning. As a proof of concept, we conditionally deleted two genes (i) the CDK related kinase CRK3, an essential gene implicated in cell cycle progression in Leishmania and (ii) PF16, a non-essential gene coding a flagellar protein. The rapidity and efficiency of this system opens up novel insights into functional characterization of essential and nonessential genes in Leishmania.

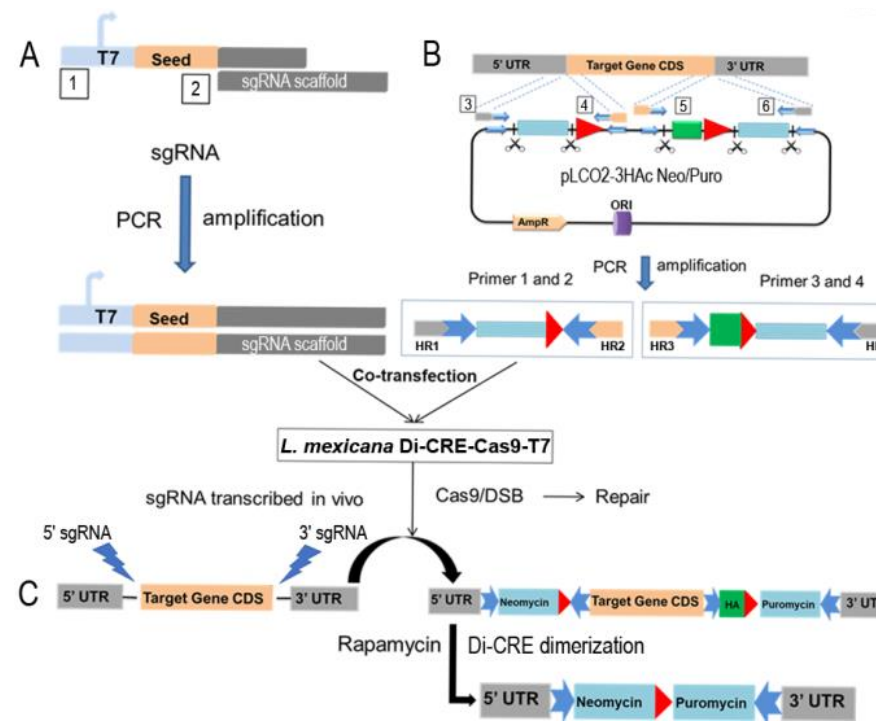

\section{Figure 1: Inducible system: the Floxed gene strategy}

(A) PCR generation of the sgRNA after annealing of two primers: the forward primer that contains the $\mathrm{T} 7$ promotor, $20 \mathrm{bp}$ specific to the target gene and beginning of the sgRNA scaffold (box 1) and the reverse primer for the sgRNA scaffold (box 2). (B) Donor DNA generated using the constructed plasmids as a template and the specific primers (boxes 3-6; more details in Supplementary Figure 1) consists in drug resistance cassettes (puromycin and neomycin resistance genes with their UTRs represented in light blue), the HA tag to be fused at the $\mathrm{N}$ - or C-terminus of the GOI and the LoxP sequences (red arrows). (C) Following cell transfection, gene edition by Cas9 allows LoxP integration. Rapamycin induction of the Di-CRE activity (Di-CRE dimerization) leads to gene excision.

\section{Experimental procedures}

\section{Parasite}

The cell line used was a $L$. mexicana (MNYC/BZ/62/M379) modified cell line with Di-CRE integrated in the ribosomal locus (Duncan et al., 2016) and hSpCas9 and T7 polymerase episomally expressed from the plasmid pTB007 (Beneke et al., 2017). L. mexicana promastigotes were cultivated at $27^{\circ} \mathrm{C}$ in HOMEM medium supplemented with $10 \%$ heat inactivated fetal calf serum (FCS) and $0.002 \%$ hemin. Transgenic cell lines were grown in the presence of appropriate antibiotics at the following concentrations: blasticidin (InvivoGen) $20 \mu \mathrm{g} \cdot \mathrm{mL}^{-1}$ and hygromycin B (InvivoGen) $50 \mu \mathrm{g} . \mathrm{mL}^{-1}$.

\section{Plasmid constructions}

For amplification of the donor DNA cassettes, two 'universal' plasmids, pLC01 and pLC02, were constructed: a sequence containing the primer linkers, the LoxP sequences, 3xHA tag sequence and the restriction sites needed were ordered as a gene block (IDT Company®). The gene blocks were received in basic backbone vectors (pUC-3HAn and pUC-3HAc) (supplementary figure1) which were amplified in the laboratory. The puromycin resistance gene was cloned with fragments from the 5'UTR (untranscribed region) of malate dehydrogenase and the 3'UTR of $60 \mathrm{~S}$ ribosomal protein (Beneke et al., 2017) between BamH1/XhoI restriction sites. The resulting vectors were than digested by SpeI and MfeI restriction enzymes to insert the neomycin resistance gene with the 5'UTR of cytochrome oxidase and 3'UTR of 3'ribosomal protein. The vectors thus generated pLC01-3HAn Puro-Neo for N-terminal tagging (Supplementary Figure 1A) and pLC02$3 \mathrm{HAc}$ Puro-Neo for C-terminal tagging (Supplementary Figure 1B) were amplified in E. coli and sequenced. Both the tag and the drug resistance sequences have 
restriction sites on each side to facilitating changing the tag or the resistance genes. These plasmids should also be usable to develop the system in Trypanosoma but changes in the drug resistance genes' UTRs may be needed.

\section{Primer design}

For this strategy, a subset of primers is needed for the guide RNA and for the donor DNA. sgRNA primers can be manually designed as described (Beneke et al., 2017). Briefly, we used a forward primer specific to the GOI which consists of a T7 promoter sequence, a 20nt sgRNA specific to the targeted site and a sequence complementary to the sgRNA scaffold and a reverse primer 'universal' for all sgRNA sequences which contains a complementary sequence for the sgRNA scaffold. The 20 nts can be designed through EuPaGDT CRISPR gRNA Design Tool (http://grna.ctegd.uga.edu/). Donor DNA primers also can be manually designed as described (Beneke \& Gluenz, 2019). These primers consist in (i) a variable part of 30 nts of a homology region specific to the target sequence and (ii) a part of 20nt termed 'linkers' which bind to the template plasmids pLC01 and pLC02, and is 'universal' for all the GOIs. Finally, the alternative for manual design is the primer design for both the sgRNA and the donor DNA automated on LeishGEdit (www.leishgedit.net), developed by Eva Gluenz's lab (Beneke et al., 2017).

\section{Primer design to preserve the UTRs of the GOI}

For this strategy, a subset of primers is needed for the guide RNA and the donor DNA. Before primer design, two regions upstream and downstream of the GOI have to be selected using transcriptomic data on TriTrypDB (https://tritrypdb.org/tritrypdb/) (Aslett et al., 2010). Then the donor DNA primers were manually designed as described previously (Beneke et al., 2017). The sequence of donor DNA primers consists in 30 nt of homology regions from each side of the target sequences next to the sgRNA binding sites and $20 \mathrm{nt}$ linkers binding to the template plasmids pLC01 or pLC02. For the sgRNA primers, the $20 \mathrm{nts}$ can be designed through EuPaGDT CRISPR gRNA Design Tool (http://grna.ctegd.uga.edu/) by uploading the sequences of the intergenic regions of the GOI; this website has the same target parameters as those used in the LeishGEdit strategy (Beneke et al., 2017) (for more details see Figure 2).

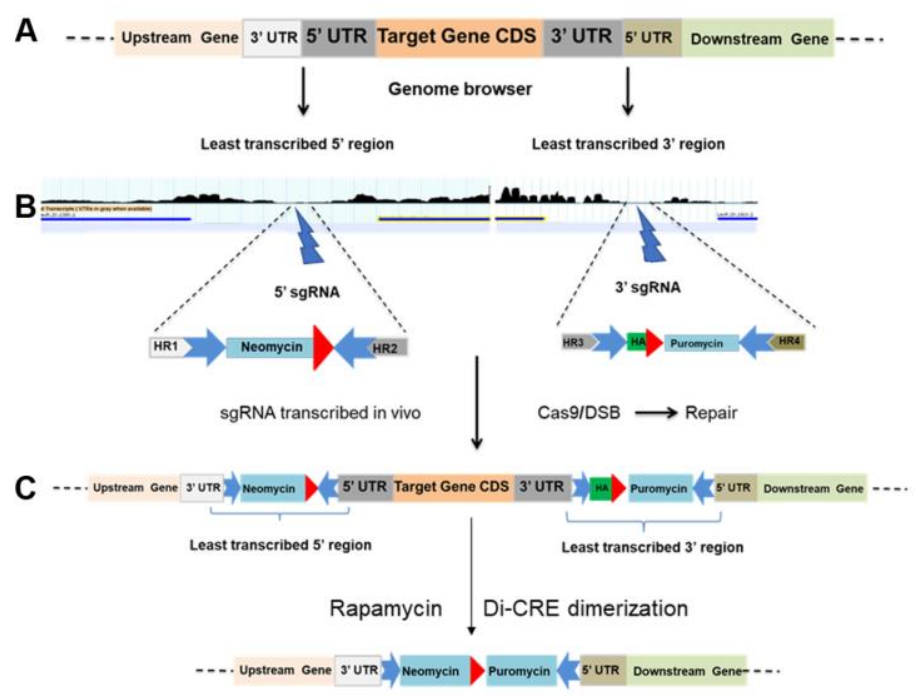

\section{Figure 2: IKO strategy preserving the UTRs}

HR: Homology region. Resistance cassettes: in light blue (including UTRs). Linkers: blue arrows. Red arrowheads: LoxP sequences. (A) Scheme of the targeted locus. (B) Selection of the least transcribed region from the 5' and 3 ' intergenic regions using genome browser on TriTrypDB (https://tritrypdb.org/tritrypdb/), and scheme of the constructs used. (C) Gene edition by the CRISPRCas9 system which allows LoxP integration, followed by gene excision after rapamycin induction of the Di-CRE activity.

\section{PCR amplification of the donor DNAs and sgRNAs}

For the amplification of the neomycin and puromycin cassettes, $20 \mathrm{ng}$ of circular pLC01 or pLC02 (depending on the presence of absence of a HA tag and the $\mathrm{N}$ or $\mathrm{C}$ terminal position of the tag) were used; $0.2 \mathrm{mM}$ dNTPs, $2 \mu \mathrm{M}$ each of genespecific forward and reverse primers, and 1 
unit Phusion Polymerase (NEB) were mixed in $1 \times$ Phusion reaction buffer, in a $50 \mu \mathrm{L}$ total volume. PCR steps were 30 s at $98^{\circ} \mathrm{C}$ followed by 35 cycles of $30 \mathrm{~s}$ at $98^{\circ} \mathrm{C}, 30 \mathrm{~s}$ at $61^{\circ} \mathrm{C}, 1 \min$ at $72^{\circ} \mathrm{C}$, followed by a final elongation step for $10 \mathrm{~min}$ at $72^{\circ} \mathrm{C} .2 \mu \mathrm{L}$ of this reaction was run on a $1 \%$ agarose gel to check for the presence of the expected product. PCR products were purified using the Kit Wizard®SV Gel and PCR Clean-Up System (Promega ${ }^{\circledR}$ ) and then heatsterilized at $75^{\circ} \mathrm{C}$ for $10 \mathrm{~min}$ and used for transfection. For amplification of sgRNAs, $0.2 \mathrm{mM}$ dNTPs, $2 \mu \mathrm{M}$ each of a reverse primer ML42 (sgRNA scaffold) and a forward primer containing the 20 nts specific to the GOI, and 1 unit Phusion Polymerase (NEB) were mixed in $1 \times$ Phusion reaction buffer, in a $50 \mu \mathrm{L}$ total volume. PCR steps were $30 \mathrm{~s}$ at $98^{\circ} \mathrm{C}$ followed by 35 cycles of $30 \mathrm{~s}$ at $98^{\circ} \mathrm{C}, 30 \mathrm{~s}$ at $60^{\circ} \mathrm{C}, 10 \mathrm{~s}$ at $72^{\circ} \mathrm{C}$, followed by a final elongation step for $10 \mathrm{~min}$ at $72^{\circ} \mathrm{C}$. PCR products were checked on a $2 \%$ agarose gel and purified as explained below.

\section{Transfection}

Parasite transfections were performed as described previously (Beneke et al., 2017). Briefly, an exponentially growing culture of a Leishmania promastigote cell line expressing Cas9 and Di-CRE was prepared. $6 \times 10^{6}$ cells were used for each transfection. PCR products (sgRNA template and donor DNA) were purified and eluted in $50 \mu \mathrm{L}$, for a total amount of $20 \mu \mathrm{g}$ per transfection $(10 \mu \mathrm{g}$ for Neomycin and 5' sgRNA and $10 \mu \mathrm{g}$ for Puromycin and 3' sgRNA). Just before transfection, $1 \mathrm{X}$ transfection buffer master mix $(1.5 \mathrm{mM}$ $\mathrm{CaCl} 2,3 \times \mathrm{Tb}-\mathrm{BSF}$ buffer) was prepared. Using the Amaxa Nucleofector $2 b$ and $X-$ 001 program. The Transfected cells were quickly shifted into prewarmed HOMEM medium. After incubation between $18 \mathrm{~h}$ and $24 \mathrm{~h}$ at $27^{\circ} \mathrm{C}$, the selection drugs were added (neomycin and puromycin). As soon as possible generally after 8-10 days post transfection, the recovered transfectants were cloned into 96-well plates. After 1015 days, the recovered clones were screened by PCRs to select $100 \%$ edited clones.

\section{Clone screening}

Total DNA was extracted with NucleoSpin ${ }^{\circledR}$ Blood QuickPure Kit (Macherey-Nagel) following the manufacturer's instructions. For verifying gene edition in the transfected lines/clones, primers were designed on each side of the GOI so as to differentiate the edited alleles from the wild-type ones. A first PCR was run using two couples of primers: one couple in the 5'UTR and another one in 3'UTR of the GOI; then more PCRs, using different primer couples, were performed on the selected clones for confirmation. A $100 \%$ edited clone was selected for further phenotypic characterization.

\section{Growth curve and cell preparation}

Cells in the early stage of exponential growth $\left(1 \times 10^{6}\right.$ cells $\left.\mathrm{mL}^{-1}\right)$ were used in all the experiments. Cells were induced with $800 \mathrm{nM}$ of rapamycin (Abcam). Each day, induced and non-induced cells were counted and re-diluted to the same number $\left(1 \times 10^{6}\right.$ cells $\left.\mathrm{mL}^{-1}\right)$. Genomic DNA extraction and cell preparation for flow cytometry analysis were performed daily to follow gene excision after Di-CRE activation.

\section{Quantitative PCR analysis of non- induced and induced cell lines}

Extraction of total DNA from non-induced (control) and rapamycin induced cells was performed as described below. For all the samples, the extracted genomic DNA concentration was measured using a Nanodrop ${ }^{\circledR}$ and diluted at 100ng $\mu \mathrm{L}^{-1}$. Quantitative PCR assays were conducted using the LightCycler-480 SYBR Green I master kit (Roche). Reaction mixtures were composed according to the manufacturer's protocol for a final volume 
of $15 \mu \mathrm{L}$. Reactions were carried out in a ROCHE Real-Time System using the following cycling conditions: $95^{\circ} \mathrm{C}$ for 5 min, followed by 44 cycles at $95^{\circ} \mathrm{C}$ for $10 \mathrm{~s}, 58^{\circ} \mathrm{C}$ for $10 \mathrm{~s}$ and $72^{\circ} \mathrm{C}$ for $10 \mathrm{~s}$. Two qPCR technical replicates were conducted for each biological replicate. CRK3 and PF16 specific primers were used to quantify gene amounts, with the flabarin gene as a control (see list of primers in Supplementary Table 1).

\section{Flow Cytometry Analysis}

A total of $2.10^{7}$ cells from mid-log phase culture of L. mexicana $\left[C R K 3^{\text {Flox }}\right.$ Puro$\mathrm{Neo}$ cells were used for each sample. Cells were washed with $10 \mathrm{~mL}$ PBS 1X, resuspended in $1 \mathrm{~mL}$ of ice-cold $70 \%$ methanol, vortexed and incubated at $+4^{\circ} \mathrm{C}$ for a maximum of 10 days. Prior to analysis, fixed cells were harvested by centrifugation at $1000 \mathrm{~g}$ for $5 \mathrm{~min}$ at $+4^{\circ} \mathrm{C}$, washed with $10 \mathrm{~mL}$ PBS 1X, and resuspended in $500 \mu \mathrm{L}$ of SYBER Green (1/5000 in PBS). Cells were gently mixed, kept $5 \mathrm{~min}$ at room temperature, and then analyzed on the fluorocytometer "Miltenyi MACS quant".

\section{Microscopy and fluorescence imaging}

Transfected cells were grown to mid-log phase, harvested and washed with PBS 1X, fixed with $4 \%$ paraformaldehyde for 30 min, washed with PBS and stored in PBS at $+4{ }^{\circ} \mathrm{C}$ until use. Cells were adhered to glass slides coated with poly-L-lysine, then neutralized with $100 \mathrm{mM}$ glycine and permeabilized with $0.2 \%$ Triton $\mathrm{X}-100$ for 10 min. Cells were blocked with $1 \%$ bovine serum albumin (BSA) in PBS for 1 h. Cells were incubated with anti-HA diluted in PBS-BSA for $1 \mathrm{~h}$. Primary antibodies were visualized with the appropriate secondary antibodies conjugated with Alexa Fluor 488. DNA was stained with Hoechst 33342 (Thermo Scientific). Finally slides were mounted with SlowFade ${ }^{\circledR}$ Gold Antifade Reagent
(Thermo Fisher-Invitrogen). Cells were viewed by phase contrast, and fluorescence was visualized using appropriate filters on a Zeiss® Axioplan 2 microscope with a $100 \times$ objective. Digital images were captured using a Photometrics CoolSnap CCD camera (Roper Scientific $®$ ) and processed with MetaView (Universal Imaging().

\section{Parasite tracking}

Leishmania promastigotes (PF16 iKO non induced and rapamycin induced) from exponential phase were mounted between slide and coverslip in culture medium. Parasites were immediately imaged to make 330 seconds films, using an inverted microscope (Zeiss AxioObserver) driven by ZEN software (Zeiss). The temperature was set up to $27^{\circ} \mathrm{C}$. We used a $10 \mathrm{X} / 0.25$ A-Plan ZEISS objective, Coolsnap HQ2 camera $1392 \times 1040$ imaging array, with pixel $=6.45 \mu \mathrm{m}$,. Image treatment was done using Fiji, tracking was performed using TrackMate pluging(Tinevez et al., 2017).

\section{Results and discussion}

\section{Rationale for an inducible gene deletion strategy combining Di- CRE and Cas9}

The recent CRISPR-Cas9 systems set up for Leishmania are adapted for studying non-essential genes, but the $\mathrm{KO}$ of essential genes requires an inducible system. In a preliminary work, we adapted the T7 inducible system from Yurchenko and Wilson laboratories (Yao et al., 2007); (Kraeva et al., 2014), using the inducible expression of Myosin Like Protein 1 fused to a C-terminal HA-tagged (MLP1-HAc); however, the rates of edited parasites were similar without and with tetracycline induction (Supplementary Figure 2A), indicating that the system was very leaky. J. Mottram's group (Duncan et al., 2016) has developed an inducible strategy based on Di-CRE for knocking out essential 
genes which was a useful initiative for the field at that time. However, we found this method cumbersome, as it needs numerous steps of molecular cloning and several rounds of transfections. The inducible CRISPR-Cas9 system that we have established combines the advantages of the CRISPR-Cas9 and the Di-CRE recombinase systems. Our system requires a Leishmania cell line expressing the DiCRE subunits, the nuclease Cas9 and the T7 RNA polymerase. We first transfected the L. mexicana Di-CRE cell line (shared by J. Mottram) with the circular plasmid pTB007 (shared by E. Gluenz) for the expression of the humanized Streptococcus pyogenes Cas9 and the T7 RNA polymerase. The sgRNA is generated and transfected as a PCR product to be transcribed in vivo by the $\mathrm{T} 7$ polymerase (Figure 1A, see also Experimental procedures for the details). The donor DNA containing the drug resistance cassettes for the selection and the LoxP sequences is also provided as a PCR product, amplified from 'universal' plasmids templates that we constructed previously (see Experimental procedures, Figure 1B and Supplementary Figure 1). The repair of the DSB at the 5' and 3' ends of the GOI allows the integration of the LoxP sequences and resistance cassettes to generate a Floxed targeted gene cell line (Figure 1C). These edited parasites are obtained after a single round of transfection, which generally takes 8-10 days, making this method highly efficient and time-saving compared to the previously published conditional $\mathrm{KO}$ method (Santos et al., 2017). In some cases, most probably because certain genes cannot tolerate UTR modifications, the strategy in which the GOI is directly flanked by LoxP sequences showed limited efficiency; and edited parasites with modified UTRs did not recover after transfection, or exhibited growth defects and/or phenotype changes before induction. We therefore wished to set up an original alternative strategy targeting intergenic regions as transcriptionally 'neutral' as possible in order to preserve the UTR sequences of the genes of interest.

\section{Conditional deletion of CRK3 affects $L$. mexicana survival}

As a proof of concept of the floxed gene strategy, we first conditionally deleted the Cyclin-related kinase 3 (CRK3) gene (Figure 3), which has previously been characterized as an essential gene in $L$. mexicana (Hassan, Fergusson, Grant, \& Mottram, 2001); (Duncan et al., 2016).

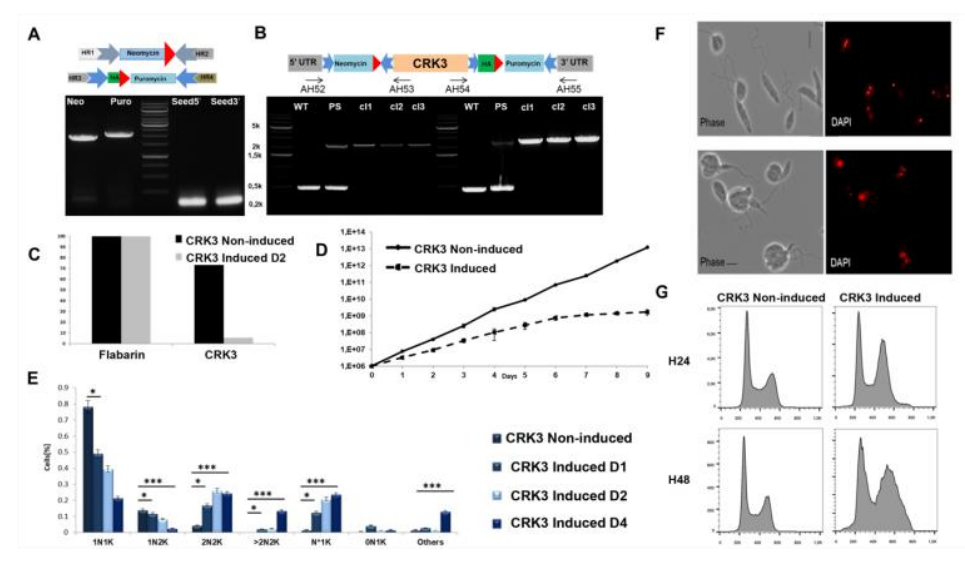
Figure 3: Phenotype analysis of CRK3
mutants.

(A) Schemes of donor DNAs (top) and agarose gel showing the size and quantity of PCR-generated donor DNAs (Neo and Puro) and sgRNAs (Seed5 and Seed3). (B) Scheme of the edited locus (top) and PCR analysis to select edited clones using primer pairs in the 5' and 3' UTRs and coding sequences of the targeted gene. PCR using primer pairs AH52:AH53 and AH54:AH55; from left to right: WT: L. mexicana Di-CRE-Cas9 strain showing a 400bp amplicon ('wild-type' control); PS: the transfected strain before cloning (parental strain) shows two PCR products, one at $400 \mathrm{bp}$ for the WT allele of CRK3 and one at $2400 \mathrm{bp}$ for the edited allele with integration of Neomycin+LoxP1 sequence on the 5' side and Puromycin+LoxP2 on the 3' side; the tested clones (cl1, cl2, cl3) with 100\% edition (no WT allele detected on both 5' and 3' sides). (C) qPCR results performed on genomic DNA extracted from a [CRK $3^{\text {Flox }}$ Puro-Neo] clone either non-induced or induced two days with rapamycin, using primers specific to CRK3 and, as a control, primers specific to the housekeeping gene flabarin. (D) Growth curves of the selected [CRK3 $3^{\text {Flox }}$ Puro-Neo] clone before (Non-induced) and after rapamycin induction. (E) Nucleus/kinetoplast patterns established after DNA labeling with DAPI, for noninduced and induced cell lines $\left(\mathrm{N}^{*} 1 \mathrm{~K}\right.$ : cells with large deformed nucleus). Significance after the Chi-square test: *: $\mathrm{p}<0.001$; ***: $\mathrm{p}<0.00001$. (F) Microscopic analysis 
before and after induction: Bright field and DAPI fluorescence (red), showing severe defects in cell morphology and deformed large nuclei corresponding to cells blocked at the $2 \mathrm{~N} 2 \mathrm{~K}$ phase. (G) Flow cytometry analysis of the selected CRK3 mutant clone before and after rapamycin induction during $24 \mathrm{~h}$ and $48 \mathrm{~h}$; left panel: non-induced; right panel: induced.

We transfected the L. mexicana Di-CRECas9 cell line with PCR products containing the sgRNA and the donor DNA with or without an HA tag (see Experimental procedures) to generate the cell lines $\left[C R K 3^{\text {Flox }}\right.$ Puro-Neo] and [CRK3$H A^{\text {Flox }}$ Puro-Neo] respectively (Figure 3 and Supplementary Figure 2B-C). After transfection, selection and cell cloning, a high edition rate was obtained for $\left[C R K 3^{\text {Flox }}\right.$ Puro-Neo], with numerous clones '100\% edited', i.e. showing no remaining wild-type copy of $C R K 3$. However, in the case of $\left[C R K 3-H A^{\text {Flox }}\right.$ Puro-Neo], the edition rate was lower, probably because of the tag that may have a negative effect on parasite fitness. Gene edition was confirmed by PCR analysis (Figure 3B). Prior to perform phenotypic characterization of CRK3 mutant cells, we confirmed that rapamycin at the concentration of $800 \mathrm{nM}$ did not affect the growth of the control cell line $L$. mexicana Di-CRE-Cas9 (Supplementary Figure 2D). Di-CRE-mediated excision of CRK3 after rapamycin induction was followed by PCR analysis each day post induction. Using specific primers flanking the $\left[C R K 3^{\text {Flox }}\right.$ Puro-Neo] construction, a $5110 \mathrm{bp}$ PCR product was amplified before rapamycin induction; while a PCR product of $4000 \mathrm{bp}$, concordant with the excised gene size, progressively appeared following rapamycin induction (Supplementary Figure 2B). This result shows that the Di-CRE activity is not leaky in the absence of rapamycin and that the system is tightly regulated. Using qPCR, we then examined the $C R K 3$ excision rates after induction, and showed that the $C R K 3$ loss was achieved at $90 \%$ from D2 post induction onwards; yet, the excision was never completely achieved, and around $10 \%$ of the gene copies persisted in the cell population (Figure 3C). These data correlate with those of Duncan et al. where a slight amount of CRK3 protein remained detectable after induction (Duncan et al., 2016). The growth rate of $\left[C R K 3^{\text {Flox }}\right.$ PuroNeo] and [CRK3-HA $A^{\text {Flox }}$ Puro-Neo] was assessed during eight days after induction (Figure 3D and Supplementary Figure 2C respectively); and a severe growth defect was observed in induced cells compared with non-induced control cells as early as D2. The nucleus/kinetoplast pattern (NK pattern) of non-induced and induced parasites showed a decrease in $1 \mathrm{~N} 1 \mathrm{~K}$ cells and a significant increase in $2 \mathrm{~N} 2 \mathrm{~K}$ cells, multinucleated cells $(>2 \mathrm{~N} 2 \mathrm{~K})$ and cells with large deformed nuclei $\left(\mathrm{N}^{*} 1 \mathrm{~K}\right)$ and abnormal cell morphology (Figure 3E, Figure 3F). These results correlated with flow cytometry analysis of the $\left[C R K 3^{\text {Flox }}\right.$ Puro-Neo] cell line before and after rapamycin induction, which showed a severe defect in DNA contents of induced parasites, with essentially an accumulation of cells blocked in G2/M (Figure 3G). Our results are in agreement with those already published for this gene and validate the efficiency of our CRISPR-Cas9 combined to Di-CRE inducible system to elucidate the function of essential genes in Leishmania in a considerably shortened time.

\section{Conditional deletion highlights that PF16 is sensitive to UTR modifications}

When an essential gene is used for a proof of concept, some deleterious events may not be noticed. So, to have more insights in our system, i.e. to be able to detect any limitations or deleterious events during parasites recovery, we decided to edit a non-essential gene, PF16, encoding a protein of the central pair of microtubules of the flagellar axoneme (Beneke et al., 2017; Martel, Beneke, Gluenz, Spath, \& Rachidi, 2017). Conditional editing of nonessential genes is also interesting, in particular to follow the early phenotypes 
secondary to protein depletion and to avoid parasite adaptation (see below). Like for the CRK3 iKO, we used LeishGedit to automatically design the sgRNA and donor DNA for PF16 iKO. We transfected the $L$. mexicana Di-CRE-Cas9 cell line with PCR products containing the sgRNAs and the donor DNAs (see Experimental procedures). After cell cloning, [PF16$H A^{\text {Flox }}$ Puro-Neo] cell lines were obtained with a high rate of edition (Supplementary Figure 3A). The follow-up of PF16 rapamycin-mediated excision by PCR analysis (Supplementary Figure 3B) showed that all PF16 alleles were excised after 10 days post-induction. Immunofluorescence confirmed a reduction of the PF16-HA signal, correctly located at the flagellum (Supplementary Figure 3C). Our results demonstrate that the system is efficient both at the genomic and protein levels for PF16 (Supplementary Figure 3C, Supplementary Figure 3D). Parasite growth was not affected after PF16 depletion (Supplementary Figure 3E), in agreement with published results in L. mexicana and L. donovani (Beneke et al., 2017; Martel et al., 2017). Surprisingly however, parasites of the $\left[P F 16-H A^{\text {Flox }}\right.$ Puro-Neo] clones were not motile even before induction. This result highlights one of the limitations of this strategy. We analyzed several mutants to clarify what, of the HA tag or UTR modifications, was responsible for this phenotype. First, the same phenomenon was observed with $\left[\right.$ PF $16^{\text {Flox }}$ Puro-Neo] parasites, i.e. without an HA tag, thus ruling out any role for the tag. Second, when modifying each UTR separately, edited parasites with a modified 3' UTR (Supplementary Figure 4A) were motile and showed a correct protein localization in immunofluorescence (Supplementary Figure 4B upper panel); whereas edited parasites with a modified 5' UTR were paralyzed and displayed a localization defect (Supplementary Figure 4A and 4B lower panel). We therefore concluded that, although PF16 is a non-essential gene, it is sensitive to UTR modifications (5' UTR). This led us to propose an alternative strategy to preserve native UTRs.

\section{UTR preservation strategy}

In Trypanosomatids, protein coding genes are present in large polycistronic units which are constitutively transcribed by Pol II (reviewed in (C. E. Clayton, 2002). In contrast to other eukaryotes, there is no evidence for transcriptional regulation. The regulation occurs mainly at the posttranscriptional level through cis- and transacting elements (C. Clayton \& Shapira, 2007). These elements have a role for example in the splicing and stability/instability of mRNAs, and/or in the efficiency of translation (Freitas Castro et al., 2017; Pasion, Hines, Ou, Mahmood, \& Ray, 1996). Taking into account the importance of endogenous UTRs for certain genes, we designed a new strategy in which, instead of closely flanking the gene with LoxP sequences, we would target the least transcribed intergenic regions (Figure 2), therefore preserving endogenous UTRs. In this strategy, we used the same parameters and template plasmids as described above to amplify the donor DNAs. The primers for sgRNA and homology regions were designed to target the least transcribed regions upstream and downstream of the GOI, selected using transcriptomic data from TriTrypDB (https://tritrypdb.org/tritrypdb/). After transfection and integration of the donor DNAs, gene excision was followed by PCR. To assess the efficiency of this second strategy we repeated the iKOs of PF16 and CRK3 described here above. We selected the least transcribed region in the intergenic regions surrounding PF16 (229bp from the PF16 ATG, and 1112bp from the stop codon) and surrounding CRK3 (580bp from CRK3 ATG, and 454bp from the stop codon) respectively. After manual design of the homology regions and sgRNA primers, the PCR products were generated and transfected into the $L$. mexicana Di-CRE-Cas9 cell line. In both 
cases (Figure 4 and Figure 5), edition of PF16 and CRK3 transfectants was rapidly obtained (day 8-10). Follow-up of PF16 (Figure 4C) and CRK3 (Figure 5B) excision after rapamycin induction by qPCR showed that the UTR preservation strategy was as efficient as the first strategy. Characterization of the phenotypes, analyzed as described above, showed identical results to those of the first strategy for CRK3 (Figure 4 and Figure 5). PF16-edited clones were motile before induction, and became paralyzed after induction (Figure 4E).

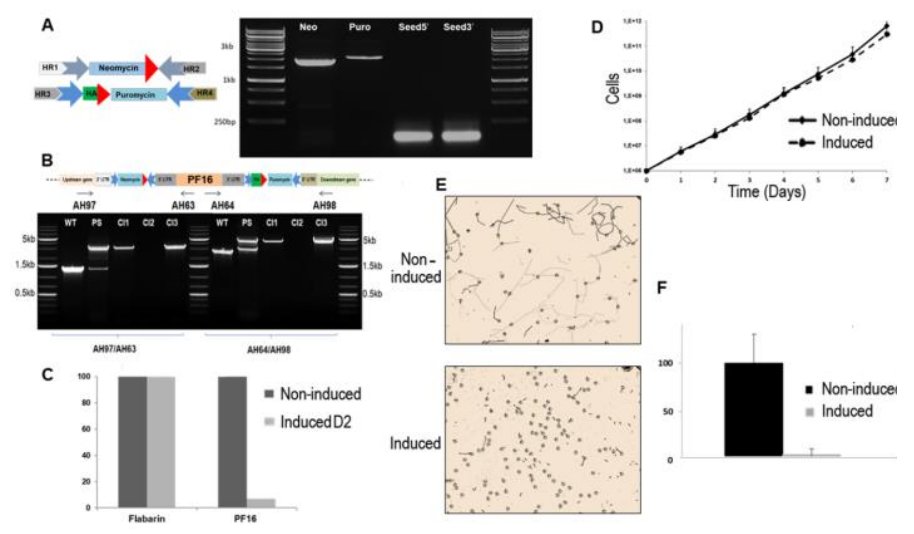

\section{Figure 4: PF16 mutant as a proof of concept for the iKO strategy preserving UTRs.}

(A) Agarose gel to check size and quantity of PCRgenerated Donor DNAs (Neo: homologous regionsneomycin cassette-LoxP1-homologous regions, Puro: homologous regions-puromycin cassette-LoxP2homologous regions, same color coding as for Figure 1) and sgRNAs (Seed 5 and seed3: 5' and 3' seeds targeting the least transcribed intergenic regions around PF16) (B) Scheme of the edited locus with preserved UTRs (top) and PCR analysis to select edited clones using primer pairs in the 5' and 3' intergenic regions (AH97:AH63 and AH98:AH64 respectively); from left to right: Primer pair AH97:AH63: WT: L. mexicana Di-CRE-Cas9 strain amplified around $1320 \mathrm{bp}$ (WT control), the transfected strain before cloning (parental strain PS) amplified two PCR products $1320 \mathrm{bp}$ for the WT allele of PF16 and the $3070 \mathrm{bp}$ for the edited allele with integration of neomycin+LoxP1 sequence in 5' and Primer pair AH98:AH64: WT: L. mexicana Di-CRE-Cas9 strain amplify around $2401 \mathrm{bp}$ (WT control), the transfected strain before cloning (parental strain PS) amplify two PCR products $2401 \mathrm{bp}$ for the WT allele of PF16 and the $4355 \mathrm{bp}$ for the edited allele with integration of puromycin+LoxP2 in the 3' side. The tested clones cl1 and $\mathrm{cl} 3$ have $100 \%$ edition and no WT allele detected in both side 5' and 3'. (C) qPCR results performed on gDNA extracted from $\left[P F 16^{\text {Flox }}\right.$ Puro-Neo] clone non- induced and induced day two post induction using primers specific to PF16 CDS and as a control primer specific to housekeeping gene flabarin. (D) Growth curves of the selected $\left[\right.$ PF $16^{\text {Flox }}$ Puro-Neo] clone before (non-induced) and after rapamycin induction. (E) Motility assay by time laps microscopy before and after induction (PF16 KO). Images captured every 30s during $5 \mathrm{~min}$; tracking of individual parasites was performed using a trackmate plugin in FIJI. (F) Histogram showing the results of parasite tracking performed in E.

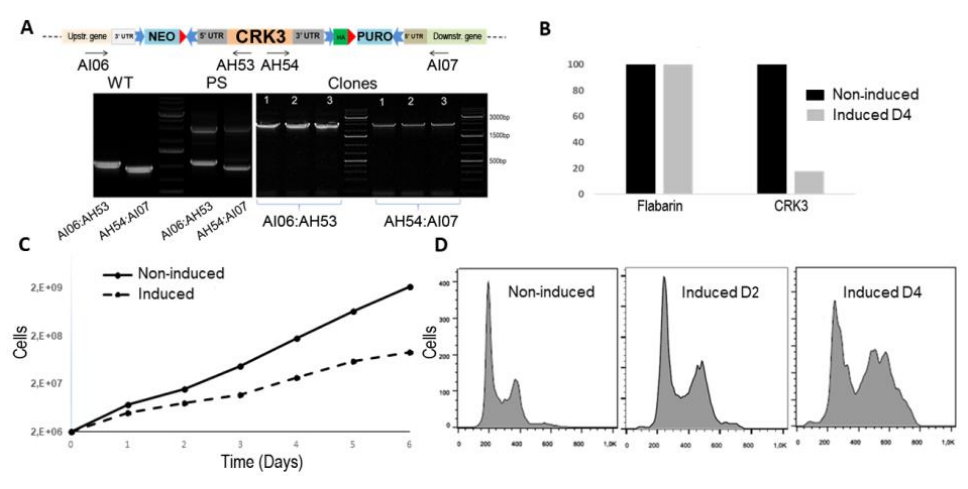

Figure 5: CRK3 mutant as another proof of concept for the iKO strategy preserving UTRs.

(A) Scheme of the edited locus with preserved UTRs (top, same color coding as for Figure 1) and PCR analysis to select edited clones using primer pairs in the 5' and 3' intergenic regions (AI06 and AI07) and in the targeted gene (AH53 and AH54); from left to right: Primer pair AI06:AH53: WT: L. mexicana Di-CRE-Cas9 strain amplify around $1100 \mathrm{bp}$ ), the transfected strain before cloning (parental strain PS) amplify two PCR products $1100 \mathrm{bp}$ for the WT allele of CRK3 and the $2995 \mathrm{bp}$ for the edited allele with integration of Neomycin+LoxP1 sequence in 5' side. Primer pair AI07:AH54: WT: $L$. mexicana Di-CRE-Cas9 strain amplify around 964 bp), the transfected strain before cloning (parental strain PS) amplify two PCR products $964 \mathrm{bp}$ for the WT allele of CRK3 and the 2935 bp for the edited allele with integration of puromycin+LoxP2 in the 3' side. The tested clones $(\mathrm{cl} 1, \mathrm{cl} 2, \mathrm{cl} 3)$ were $100 \%$ edited with no WT allele detected in both ends'. (C) qPCR results performed on gDNA extracted from CRK $3^{\text {Flox }}$ Puro-Neo clone non-induced and induced using primers specific to CRK3 CDS and as a control primer specific to housekeeping gene flabarin. (D) growth curves of the selected [CRK $3^{\text {Flox }}$ Puro-Neo] clone before (non-induced) and after rapamycin induction (E) Flow cytometry analysis of CRK3 mutant cell before and after rapamycin induction.

\section{Useful tips for designing a strategy for one's favorite GOI}

The main difficulty in editing genes in Leishmania is the high genomic plasticity that leads to gene duplication or even whole chromosome amplification, hence 
the persistence of a wild-type copy of the targeted gene. Therefore, to obtain a $100 \%$ edited cell line, a cell cloning step is generally necessary. To date, our system is highly efficient as judged by the set of iKOs which were generated in the laboratory. However, this efficiency is variable, depending on many parameters including the targeted gene itself, addition of a tag, etc. Thus, in the iKO of CRK3, the efficiency, measured as the rate of $100 \%$ edited cell clones, was 0/24 when an $\mathrm{HA}$ tag was positioned in $\mathrm{N}$-terminal position, 1/24 with the $\mathrm{HA}$ tag in $\mathrm{C}$ terminal position, and 12 and 24/24 for two attempts without any tag. Similarly, the figures for PF16 ranged from 4/24 to $24 / 24$. Using a strategy with a tag can be useful to localize the GOI before inducing the $\mathrm{KO}$ without the need for extra transfections. More importantly, the tag can be used to follow protein depletion by immunofluorescence and western blot after inducing the KO. In our experience, tagging the $\mathrm{C}$-terminus is generally safer than tagging the N-terminus in Leishmania. This is contrary to what is known in T. brucei where the tag is best positioned at the N-terminal end (Vasquez, Wedel, Cosentino, \& Siegel, 2018). However, this has to be tempered and likely depends on the targeted gene either due to the presence of targeting motifs which have a pivotal role in the protein localization and addressing to specific cellular compartment and/or to the role of the endogenous UTR in posttranscriptional regulation. A pitfall when designing a $\mathrm{KO}$ strategy is to provide sequences of donor DNA that can act as homology regions, which in turn reduces efficiency and may yield undesired recombination events. At the most, it is possible to obtain a Floxed gene with a donor DNA made of the entire gene sequence; but one cannot avoid undesirable recombination events due to this large sequence of homology. An alternative is to provide a recodonized version of the targeted gene. However, it may be difficult to obtain a good PCR product amount for such a large fragment (i.e. the gene sequence, UTR sequences and gene resistance sequence). LoxP sequences which are long enough (34 bp), made of tandem repeats and provided twice in the donor DNA, can also act as homology regions. We experienced this with PF16 and obtained either parasites with a single LoxP sequence in their genome or parasites which had deleted the gene of interest in the population recovering from transfection even before induction (see clone 2 Figure 4B). Finally, we suggest to transfect the donor DNAs as two PCR products with two drug resistance markers to enhance the chances to edit all the alleles and select only clones with the correct integration.

In the present work, we have developed a powerful tool for analyzing the function of both essential and non-essential genes. Although it is possible to KO non-essential genes using classical methods, it may be preferable to use an inducible strategy for two reasons. (i) It helps preventing the problem of a potential adaptation of the KO cell line in culture. Indeed, certain genes appear 'partly essential' but not lethal, in that sense that $\mathrm{KO}$ parasites recover slowly after transfection and then regain normal growth due to some sort of compensation of the gene deletion. (ii) It is also useful to refine the phenotype analysis in case of complex phenotypes observed in cell lines recovering from a $\mathrm{KO}$, which usually correspond to a 'final' phenotype resulting from the addition of direct and indirect effects. Observing the phenotypes just after induction may be highly informative in such cases, like RNAi in other organisms. The inducible strategy developed here for $L$. mexicana is applicable in other Leishmania sp. Expressing T7, Cas9 and Di-CRE and should be applicable to T. brucei. Although the RNAi machinery is available in $T$. bruce $i$, RNAi is limited by a generally partial reduction of the target transcript, often around 50-60\% (Albisetti et al., 
2017; Dauchy et al., 2016). The remaining protein level can be functionally sufficient and makes phenotypic characterization difficult. In our system, >80\% gene depletion was observed after only two days post-induction.

In total, the approach we propose here enables, for the first time to our knowledge, the study of the function of essential genes in Leishmania using 'universal' constructions in short times. It is also highly convenient to study non-essential genes. To study an uncharacterized gene, we preconize to produce two mutants, both bearing a tag and both with one preserved UTR, i.e. inserting the first LoxP at one end of the targeted gene and the second LoxP in a 'transcriptionally neutral' part of the intergenic region on the opposite side. This allows (i) obtaining the localization of the protein with an $\mathrm{N}$ - and $\mathrm{C}$-terminal tag before inducing the $\mathrm{KO}$ and (ii) following the protein depletion by immunofluorescence and/or western blot.

\section{Acknowledgements}

We wish to acknowledge Jeremy Mottram (Univ. of York, UK), Eva Gluenz and Tom Beneke (Univ. of Oxford, UK), Vyacheslav Yurchenko (Ostravská University, the Czech Republic), C. Yao (Ross University School of Veterinary Medicine, St. Kitts \& Nevis, West Indies, USA) and Mary E. Wilson (University of Iowa, IA, USA) for generously sharing reagents, plasmids and/or cell lines. We are grateful to Elodie Jublanc and Vicki Diakou (MRI-DBS UM microscopy facility) for their technical help for microscopy, in particular for the time-lapse experiments. We also thank the EuPathDB (https://eupathdb.org/eupathdb/) team, without which this study would not have been possible. Finally, we are grateful to Kai Wengelnick (LPHI, University of Montpellier), Mila R. Corrales, Slavica Stanojcic and Maude Lévêque (MiVEGEC, University of Montpellier) for helpful discussion.

\section{Funding}

This work was supported by the Agence Nationale de la Recherche (ANR) within the frame of the "Investissements d'avenir" program [ANR 11-LABX-0024-01 "ParaFrap"]; the Centre National de la Recherche Scientifique (CNRS); the University of Montpellier, and the Centre Hospitalier Universitaire of Montpellier. AY was recipient of a grant from the French Parasitology Consortium "ParaFrap" [ANR 11-LABX-0024-01].

\section{Conflict of interest}

The authors have no conflict of interest to declare

\section{References}

Akhoundi, M., Kuhls, K., Cannet, A., Votypka, J., Marty, P., Delaunay, P., \& Sereno, D. (2016). A Historical Overview of the Classification, Evolution, and Dispersion of Leishmania Parasites and Sandflies. PLoS Negl Trop Dis, 10(3), e0004349. doi:10.1371/journal.pntd.0004349

Albisetti, A., Florimond, C., Landrein, N., Vidilaseris, K., Eggenspieler, M., Lesigang, J., . . . Bonhivers, M. (2017). Interaction between the flagellar pocket collar and the hook complex via a novel microtubulebinding protein in Trypanosoma brucei. PLoS Pathog, 13(11), e1006710. doi:10.1371/journal.ppat.1006710

Aslett, M., Aurrecoechea, C., Berriman, M., Brestelli, J., Brunk, B. P., Carrington, M., . . . Wang, H. (2010).
TriTrypDB: a functional genomic resource for the Trypanosomatidae. Nucleic Acids Res, 38(Database issue), D457-462. doi:10.1093/nar/gkp851

Beneke, T., \& Gluenz, E. (2019). LeishGEdit: A Method for Rapid Gene Knockout and Tagging Using CRISPRCas9. Methods Mol Biol, 1971, 189-210. doi:10.1007/978-1-4939-9210-2_9

Beneke, T., Madden, R., Makin, L., Valli, J., Sunter, J., \& Gluenz, E. (2017). A CRISPR Cas9 high-throughput genome editing toolkit for kinetoplastids. $R$ Soc Open Sci, 4(5), 170095. doi:10.1098/rsos.170095

Clayton, C., \& Shapira, M. (2007). Post-transcriptional regulation of gene expression in trypanosomes and 
leishmanias. Mol Biochem Parasitol, 156(2), 93-101. doi:10.1016/j.molbiopara.2007.07.007

Clayton, C. E. (2002). Life without transcriptional control? From fly to man and back again. $E M B O \mathrm{~J}$, 21(8), 1881-1888. doi:10.1093/emboj/21.8.1881 [doi]

Damasceno, J. D., Tosi, L. R. O., Santos, R., \& Mottram, J. C. (2019). DiCre-Based Inducible Gene Expression. Methods Mol Biol, 1971, 225-235. doi:10.1007/9781-4939-9210-2_11

Dauchy, F. A., Bonhivers, M., Landrein, N., Dacheux, D., Courtois, P., Lauruol, F., . . Robinson, D. R. (2016). Trypanosoma brucei CYP51: Essentiality and Targeting Therapy in an Experimental Model. PLOS Negl Trop Dis, 10(11), e0005125. doi:10.1371/journal.pntd.0005125

Duncan, S. M., Myburgh, E., Philipon, C., Brown, E., Meissner, M., Brewer, J., \& Mottram, J. C. (2016). Conditional gene deletion with DiCre demonstrates an essential role for CRK3 in Leishmania mexicana cell cycle regulation. Mol Microbiol, 100(6), 931-944. doi:10.1111/mmi.13375

Freitas Castro, F., Ruy, P. C., Nogueira Zeviani, K., Freitas Santos, R., Simoes Toledo, J., \& Kaysel Cruz, A. (2017). Evidence of putative non-coding RNAs from Leishmania untranslated regions. Mol Biochem Parasitol, 214, 69-74. doi:10.1016/j.molbiopara.2017.04.002

Hassan, P., Fergusson, D., Grant, K. M., \& Mottram, J. C. (2001). The CRK3 protein kinase is essential for cell cycle progression of Leishmania mexicana. $\mathrm{Mol}$ Biochem Parasitol, 113(2), 189-198.

lourov, I. Y., Vorsanova, S. G., \& Yurov, Y. B. (2008). Chromosomal mosaicism goes global. $\mathrm{Mol}$ Cytogenet, 1, 26. doi:1755-8166-1-26 [pii]10.1186/1755-8166-1-26 [doi]

Kraeva, N., Ishemgulova, A., Lukes, J., \& Yurchenko, V. (2014). Tetracycline-inducible gene expression system in Leishmania mexicana. Mol Biochem Parasitol, 198(1), 11-13. doi:10.1016/j.molbiopara.2014.11.002

Lye, L. F., Owens, K., Shi, H., Murta, S. M., Vieira, A. C., Turco, S. J., . . Beverley, S. M. (2010). Retention and loss of RNA interference pathways in trypanosomatid protozoans. PLoS Pathog, 6(10), e1001161. doi:10.1371/journal.ppat.1001161

Martel, D., Beneke, T., Gluenz, E., Spath, G. F., \& Rachidi, N. (2017). Characterisation of Casein Kinase 1.1 in Leishmania donovani Using the CRISPR Cas9 Toolkit. Biomed Res Int, 2017, 4635605. doi:10.1155/2017/4635605

Pasion, S. G., Hines, J. C., Ou, X., Mahmood, R., \& Ray, D. S. (1996). Sequences within the $5^{\prime}$ untranslated region regulate the levels of a kinetoplast DNA topoisomerase mRNA during the cell cycle. Mol Cell Biol, 16(12), doi:10.1128/mcb.16.12.6724
Prieto Barja, P., Pescher, P., Bussotti, G., Dumetz, F., Imamura, H., Kedra, D., . . . Spath, G. F. (2017). Haplotype selection as an adaptive mechanism in the protozoan pathogen Leishmania donovani. Nat Ecol Evol, 1(12), 1961-1969. doi:10.1038/s41559-0170361-x

Santos, R., Silva, G. L. A., Santos, E. V., Duncan, S. M., Mottram, J. C., Damasceno, J. D., \& Tosi, L. R. O. (2017). A DiCre recombinase-based system for inducible expression in Leishmania major. $\mathrm{Mol}$ Biochem Parasitol, 216, 45-48. doi:10.1016/j.molbiopara.2017.06.006

Sollelis, L., Ghorbal, M., MacPherson, C. R., Martins, R. M., Kuk, N., Crobu, L., . . . Sterkers, Y. (2015). First efficient CRISPR-Cas9-mediated genome editing in Leishmania parasites. Cell Microbiol, 17(10), 14051412. doi:10.1111/cmi.12456

Sterkers, Y., Lachaud, L., Crobu, L., Bastien, P., \& Pagès, M. (2011). FISH analysis reveals aneuploidy and continual generation of chromosomal mosaicism in Leishmania major. Cell Microbiol, 13(2), 274-283. doi:10.1111/j.1462-5822.2010.01534.x

Tinevez, J. Y., Perry, N., Schindelin, J., Hoopes, G. M., Reynolds, G. D., Laplantine, E., . . . Eliceiri, K. W. (2017). TrackMate: An open and extensible platform for single-particle tracking. Methods, 115, 80-90. doi:10.1016/j.ymeth.2016.09.016

Torres, E. M., Sokolsky, T., Tucker, C. M., Chan, L. Y., Boselli, M., Dunham, M. J., \& Amon, A. (2007). Effects of aneuploidy on cellular physiology and cell division in haploid yeast. Science, 317(5840), 916924. doi:317/5840/916 [pii]10.1126/science.1142210 [doi]

Ubeda, J. M., Legare, D., Raymond, F., Ouameur, A. A., Boisvert, S., Rigault, P., . . . Ouellette, M. (2008). Modulation of gene expression in drug resistant Leishmania is associated with gene amplification, gene deletion and chromosome aneuploidy. Genome Biol, 9(7), R115. doi:gb-2008-9-7-r115 [pii]10.1186/gb-2008-9-7-r115 [doi]

Vasquez, J. J., Wedel, C., Cosentino, R. O., \& Siegel, T. N. (2018). Exploiting CRISPR-Cas9 technology to investigate individual histone modifications. Nucleic Acids Res, 46(18), e106. doi:10.1093/nar/gky517

Yao, C., Luo, J., Hsiao, C. H., Donelson, J. E., \& Wilson, M. E. (2007). Leishmania chagasi: a tetracyclineinducible cell line driven by T7 RNA polymerase. Exp Parasitol, 116(3), 205-213. doi:10.1016/j.exppara.2007.01.001

Zhang, W. W., Lypaczewski, P., \& Matlashewski, G. (2017). Optimized CRISPR-Cas9 Genome Editing for Leishmania and Its Use To Target a Multigene Family, Induce Chromosomal Translocation, and Study DNA Break Repair Mechanisms. mSphere, 2(1). doi:10.1128/mSphere.00340-16

Zhang, W. W., \& Matlashewski, G. (2015). CRISPR-Cas9Mediated Genome Editing in Leishmania donovani. MBio, 6(4), e00861. doi:10.1128/mBio.00861-15 


\section{Supplementary material}

\section{Supplementary Table 1: List of primers}

\begin{tabular}{|c|c|c|c|c|}
\hline Name & orientation & séquence $\left(5^{\prime}-3^{\prime}\right)$ & target & purpose \\
\hline CRK3 & & & & \\
\hline \multicolumn{5}{|c|}{ UTR modified } \\
\hline AH46 & forward & TTTGTAAGGAGAGGAGACGAAGGAATTCCTGTATAATGCAGACCTGCTGC & $\mathrm{pLC02}$ & \multirow{7}{*}{$\begin{array}{l}\text { UTR modified CRK3 : Donor } \\
\text { DNAs and sgRNAs }\end{array}$} \\
\hline AH47 & reverse & GCGGGCGGTCACACGGCCAAACGAAGACATACTACCCGATCCTGATCCAG & $\mathrm{pLC} 02$ & \\
\hline $\mathrm{AH} 48$ & forward & $\begin{array}{l}\text { GAAATTAATACGACTCACTATAGGCCGAAACCTTCAAAAAAGAAGTTTTAGAG } \\
\text { CTAGAAATAGC }\end{array}$ & & \\
\hline $\begin{array}{l}\text { universal } \\
\text { sgRNArev }\end{array}$ & reverse & $\begin{array}{l}\text { AAAAGCACCGACTCGGTGCCACTTTTTCAAGTTGATAACGGACTAGCCTTATTT } \\
\text { TAACTTGCTATTTCTAGCTCTAAAAC }\end{array}$ & & \\
\hline $\mathrm{AH} 49$ & forward & CAGCACCCGTGGTTCAGCGACCTTCGTTGGGGTTCTGGTAGTGGTTCCGG & $\mathrm{pLC} 02$ & \\
\hline AH50 & reverse & TAACAAAGCACCCTCAGAAAAACAAATCCTCCAATTTGAGAGACCTGTGC & pLC02 & \\
\hline AH51 & forward & $\begin{array}{l}\text { GAAATTAATACGACTCACTATAGGCCATCGTTCAACGCGTCAGAGTTTTAGAG } \\
\text { CTAGAAATAGC }\end{array}$ & & \\
\hline AH52 & forward & GCACGTTTCTGGCCCTTTCTCC & CRK3 5'UTR & \multirow{4}{*}{ edition verification } \\
\hline AH53 & reverse & GCCTCCACATACTCGAAGACAAGG & CRK3 CDS & \\
\hline AH54 & forward & GCGACGTATTTCCCAAGTGGACC & CRK3 5'CDS & \\
\hline AH55 & reverse & GGCATGTCCAAATCTGTTTCGC & CRK3 3'UTR & \\
\hline AH85 & forward & CTGATTACCGCGACGTAT & CRK3 CDS & \multirow{2}{*}{$\mathrm{qPCR}$} \\
\hline AH86 & reverse & CGTGGATCGTACTTGAGC & CRK3 CDS & \\
\hline \multicolumn{5}{|c|}{ UTR preserved } \\
\hline AH99 & forward & CAAACACTAGTCAAAAAGACGGTGGCTGTAGTATAATGCAGACCTGCTGC & $\mathrm{pLC02}$ & \multirow{7}{*}{$\begin{array}{l}\text { UTR preserved: Donor DNAs } \\
\text { and sgRNAs }\end{array}$} \\
\hline AI01 & reverse & CTTTGCTTTACTGTCACTCGCACGAGCAGCACTACCCGATCCTGATCCAG & $\mathrm{pLC} 02$ & \\
\hline $\mathrm{AIO2}$ & forward & $\begin{array}{l}\text { GAAATTAATACGACTCACTATAGGACTGGCAGCAGCGATTTGGCGTTTTAGAG } \\
\text { CTAGAAATAGC }\end{array}$ & $\mathrm{pLC02}$ & \\
\hline $\begin{array}{l}\text { universal } \\
\text { sgRNArev }\end{array}$ & reverse & $\begin{array}{l}\text { AAAAGCACCGACTCGGTGCCACTTTTTCAAGTTGATAACGGACTAGCCTTATTT } \\
\text { TAACTTGCTATTTCTAGCTCTAAAAC }\end{array}$ & & \\
\hline $\mathrm{AI03}$ & forward & CCTTCGAGTACAGACGCCAGCTTCTTTCTCGGTTCTGGTAGTGGTTCCGG & $\mathrm{pLC} 02$ & \\
\hline $\mathrm{AI04}$ & reverse & GGCAGCTGTCAAAGTCTGTGTGTAAGCTCCCCAATTTGAGAGACCTGTGC & $\mathrm{pLC} 02$ & \\
\hline AI05 & forward & $\begin{array}{l}\text { GAAATTAATACGACTCACTATAGGATAAAGGCATGGGCTGACTCGTTTTAGAG } \\
\text { CTAGAAATAGC }\end{array}$ & $\mathrm{pLC} 02$ & \\
\hline
\end{tabular}




\begin{tabular}{|c|c|c|c|c|}
\hline AI06 & forward & TTCAGTTCGCATTGGAGCGG & $\begin{array}{l}\text { CRK3 } \\
\text { upstream gene }\end{array}$ & \multirow[b]{2}{*}{ edition check } \\
\hline AI07 & reverse & TCTGCCACCTTCACAGCAGC & $\begin{array}{l}\text { CRK3 } \\
\text { downstream } \\
\text { gene }\end{array}$ & \\
\hline \multicolumn{5}{|l|}{ PF16 } \\
\hline \multicolumn{5}{|c|}{ UTR modified } \\
\hline AH56 & forward & CCGCGCTACACAGTGGCCCTCTTCCCTCCTGTATAATGCAGACCTGCTGC & pLC02 & \multirow{7}{*}{$\begin{array}{l}\text { UTR modified PF16: Donor } \\
\text { DNAs and sgRNAs }\end{array}$} \\
\hline AH57 & reverse & GAAGGTTTGCAGAATAACCCGATTCGACATACTACCCGATCCTGATCCAG & $\mathrm{pLC} 02$ & \\
\hline AH58 & forward & $\begin{array}{l}\text { GAAATTAATACGACTCACTATAGGCTTTGTTTGGCGGACGGGAGGTTTTAGAG } \\
\text { CTAGAAATAGC }\end{array}$ & & \\
\hline $\begin{array}{l}\text { universal } \\
\text { sgRNArev }\end{array}$ & reverse & $\begin{array}{l}\text { AAAAGCACCGACTCGGTGCCACTTTTTCAAGTTGATAACGGACTAGCCTTATTT } \\
\text { TAACTTGCTATTTCTAGCTCTAAAAC }\end{array}$ & & \\
\hline AH59 & forward & AAGATCGAGAACTACCACGTGCAGCAGCACGGTTCTGGTAGTGGTTCCGG & $\mathrm{pLC02}$ & \\
\hline AH60 & reverse & CGAGCAGCGTGCATGGGCGTGACTGTGCCGCCAATTTGAGAGACCTGTGC & $\mathrm{pLC} 02$ & \\
\hline AH61 & forward & $\begin{array}{l}\text { GAAATTAATACGACTCACTATAGGCGGATGCTCAGCGGGCCTTTGTTTTAGAG } \\
\text { CTAGAAATAGC }\end{array}$ & & \\
\hline AH62 & forward & CGCTGCAGAAGTTATCCTACTCC & PF16 5'UTR & \multirow{4}{*}{ edition verification } \\
\hline AH63 & reverse & GCTCTCCTTCACGGTCGGATC & PF16 CDS & \\
\hline AH64 & forward & GCTTCCTCGTCTGTTGGACG & PF16 5'CDS & \\
\hline AH65 & reverse & GCAGAGCAGGCAATGACCGG & PF16 3'UTR & \\
\hline AH66 & forward & GAGTCCATCCAGATCATCAACA & PF16 CDS & \multirow{2}{*}{ qPCR } \\
\hline AH67 & reverse & GGTAGTTCTCGATCTTCTCGATAAA & PF16 CDS & \\
\hline \multicolumn{5}{|c|}{ UTR preserved } \\
\hline AH77 & forward & GAGGAATAGCACGTCGCTCTCTCTCTGCCCGTATAATGCAGACCTGCTGC & pLC02 & \multirow{7}{*}{$\begin{array}{l}\text { UTR preserved: Donor DNAs and } \\
\text { sgRNAs }\end{array}$} \\
\hline AH78 & reverse & AGAGACAACGATCTGCAGGGTGGCAAGCGGACTACCCGATCCTGATCCAG & $\mathrm{pLC} 02$ & \\
\hline AH79 & forward & $\begin{array}{l}\text { GAAATTAATACGACTCACTATAGGGCGGAGTACAGCACCTAGAAGTTTTAGAG } \\
\text { CTAGAAATAGC }\end{array}$ & pLC02 & \\
\hline $\begin{array}{l}\text { universal } \\
\text { sgRNArev }\end{array}$ & reverse & $\begin{array}{l}\text { AAAAGCACCGACTCGGTGCCACTTTTTCAAGTTGATAACGGACTAGCCTTATTT } \\
\text { TAACTTGCTATTTCTAGCTCTAAAAC }\end{array}$ & & \\
\hline AH80 & forward & GCGGTATACTTCTGTGCGTGCGGCGCTAGTGGTTCTGGTAGTGGTTCCGG & pLC02 & \\
\hline AH81 & reverse & GTTGACACGAGCACCTGTCACTGTGCCAGCCCAATTTGAGAGACCTGTGC & pLC02 & \\
\hline AH82 & forward & $\begin{array}{l}\text { GAAATTAATACGACTCACTATAGGGATGAGACACCGAGGCACCAGTTTTAGAG } \\
\text { CTAGAAATAGC }\end{array}$ & $\mathrm{pLC} 02$ & \\
\hline
\end{tabular}




\begin{tabular}{|l|l|l|l|l|} 
AH97 & forward & GAGCCCGCATGAACGAGC & $\begin{array}{l}\text { PF16 upstream } \\
\text { gene }\end{array}$ & \\
\hline & & & $\begin{array}{l}\text { PF16 } \\
\text { downstream } \\
\text { gene }\end{array}$ & edition check \\
AH98 & reverse & CTCCGGCACCATCGTCAAGG & \\
\hline
\end{tabular}



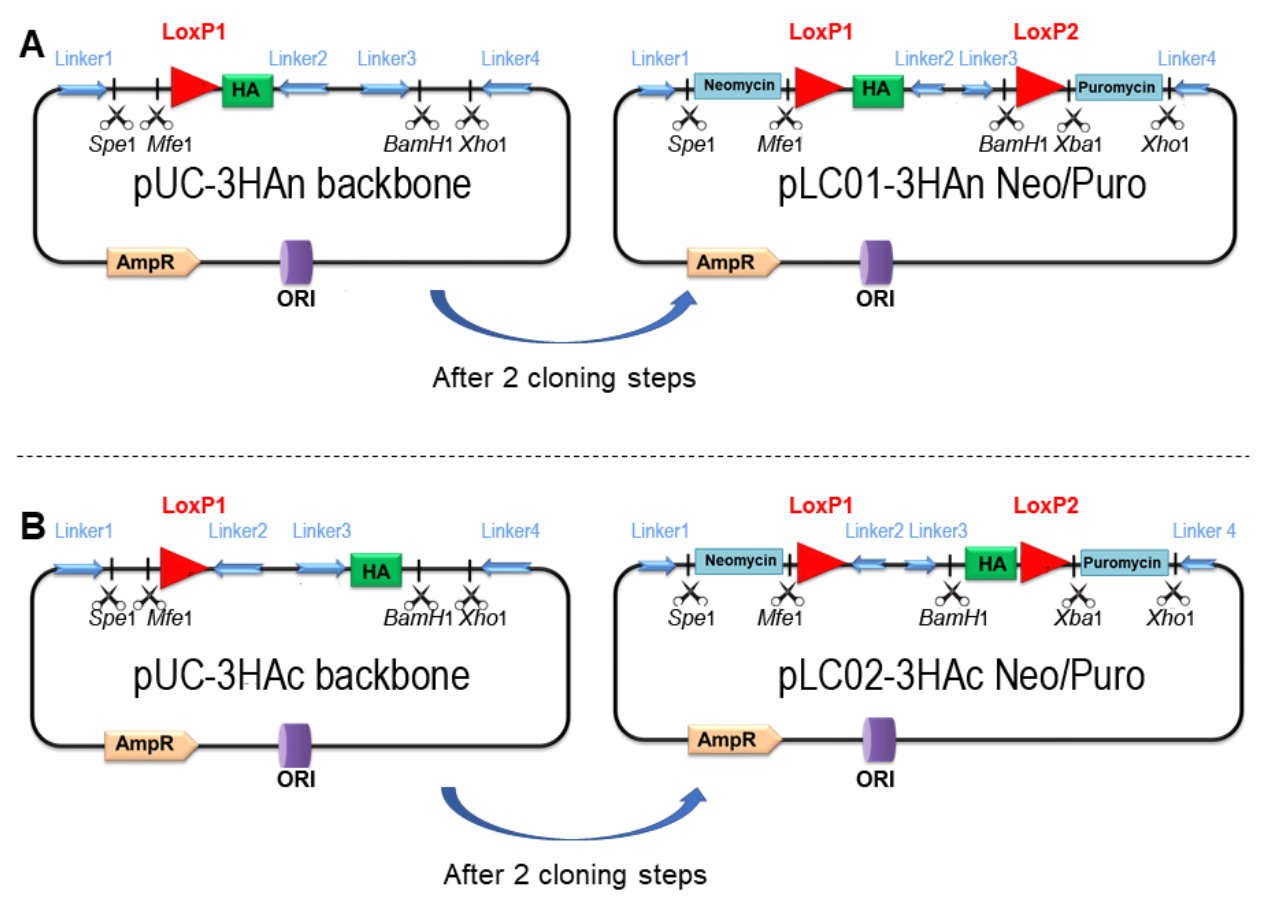

\section{Supplementary Figure 1: The 'universal' template plasmids}

Key features of the pLC01-3HAn Neo/Puro and pLC02-3HAc Neo/Puro template plasmids. HA tag (in green): 3 HA sequences to be inserted either at the N- (A) or C-terminus (B) of the GOI. Linker1-4 short sequences (Beneke et al., 2017) are those which make the plasmids 'universal'. These 'universal' template plasmids are used to obtain the donor DNA by PCR, i.e to repair the DSB. The donor DNAs consist of and to introduce the resistance cassettes Neomycin and Puromycin (including their UTRs, in light blue), \pm HA Ttag (green box) and the LoxP sequences (red arrows). The same color code is used all over the figures and supplementary figures.
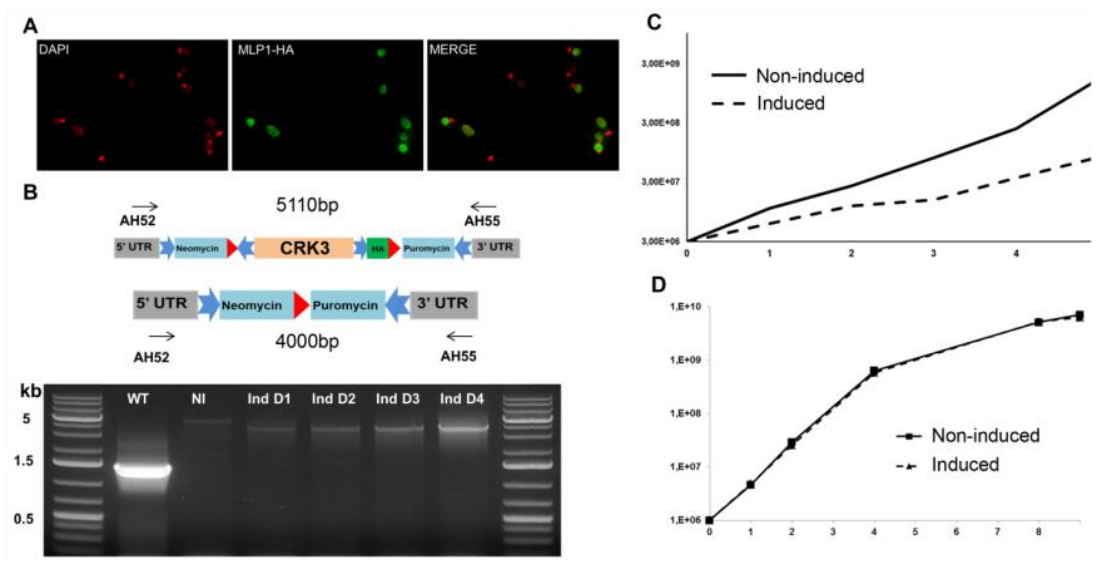

\section{Supplementary Figure 2: T7TR system is leaky and phenotypic characterization of CRK3 mutants}

(A) MLP1-HAc tagging with the T7TR system before induction: a high percentage of labelled cells was observed before tetracycline induction, showing that the system was leaky. (B) Scheme of the edited CRK3 locus with preserved UTRs (left, same color coding as for Figure 1) and PCR products using specific primers flanking the whole CRK3 edited locus AH52 and AH55; WT: wild-type allele at $1100 \mathrm{bp}$; NI: PCR product at $5110 \mathrm{bp}$ in the edited non-induced cell line; IndD1/2/3/4: PCR product at $4000 \mathrm{bp}$ corresponding to the CRK3excised locus in the induced cell line, increasing in amount from D1 to D4 (C) Growth curves of the clone F10 of CRK3-HA before (Non-induced) and after (Induced) rapamycin induction. (D) Growth curves of the $L$. mexcicana Di-CRE-Cas9 cell line after rapamycin induction (800nM): no growth defect was observed. 

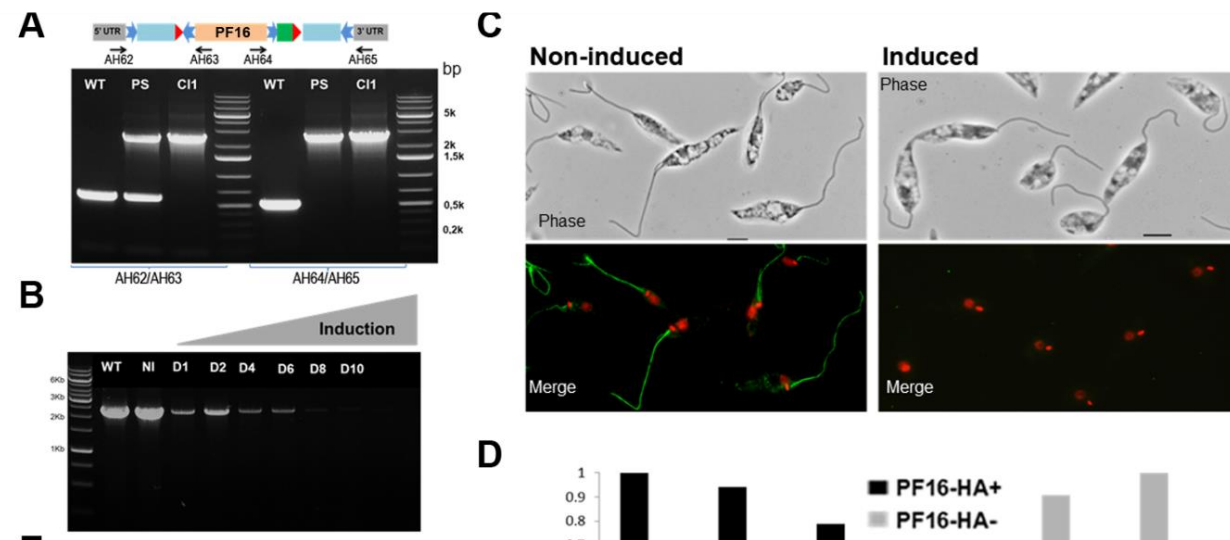

D
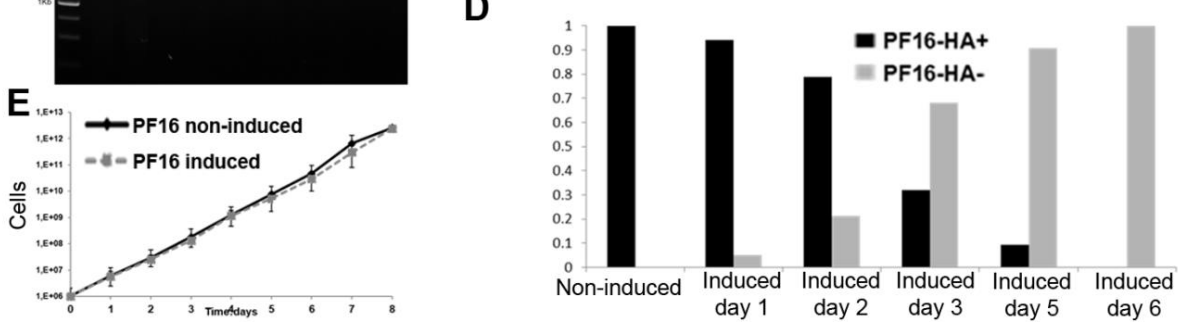

\section{Supplementary Figure 3: PF16 mutants as another proof of concept for the Floxed gene strategy}

(A) Scheme of the edited locus of interest an HA tag was positioned on the 3' side (top, same color coding as for Figure 1.) and PCR analysis to select edited clones using primers from the 5' side of PF16 (AH62:AH63) and 3' side (AH64:AH65); from left to right: WT, PS and clone 1 (cl1): one tested clone showing 100\% edition rate (no WT allele detected on both the 5' and 3' sides). (B) PCR analysis performed on genomic DNA extracted at different time points post-induction using PF16-specific primers: a decrease in PF16 amplification was observed after induction, followed by a complete extinction of the signal at day 8-10 post-induction. (C) Immunofluorescence of a PF16-HA clone (100\% edited) using an anti-HA antibody before (Non-induced) and after (Induced) rapamycin induction. (D) PF16 signal quantification based on immunofluorescence performed at different time points post-induction; >100 parasites were counted for each time point and classified as positive or negative labeling; at D8 post-induction, no PF16 labeling could be observed. (E) Growth curves of the selected [PF16-HA ${ }^{\text {Flox }}$ Puro-Neo] clone before (non-induced) and after rapamycin induction: no growth defect was observed after induction.
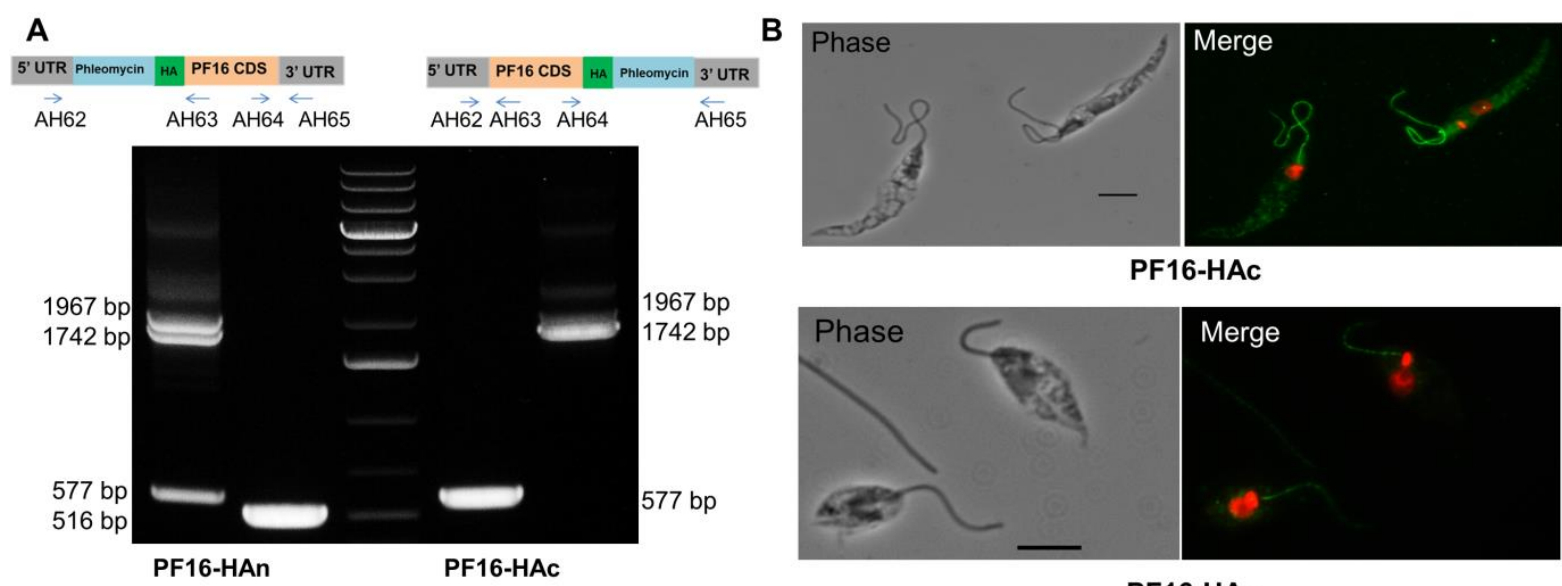

PF16-HAc

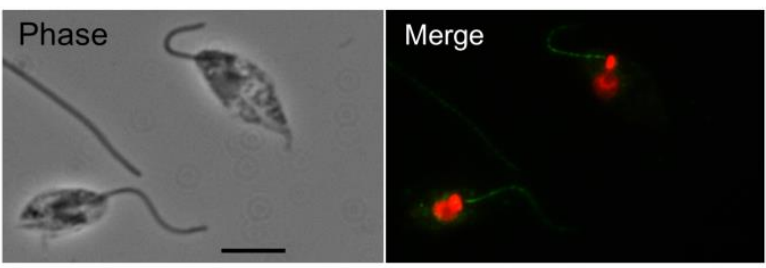

PF16-HAn

\section{Supplementary Figure 4: Phenotypic characterization of PF16 mutants}

(A) Scheme of the locus of interest (top, same color coding as for Figure 1.) and PCR analysis on PF16-HA tagged parasites with two drug resistances phleomycin and puromycin (WT band 577bp on 5' and 516 bp on 3', edited size (1742bp, 1967bp) (from left) N-terminal tagging and C-terminal. (B) Immunofluorescence with an anti-HA antibody done on PF16 parasites tagged on C-terminal (upper panel) or N-terminal (lower panel). 